\section{Genome-wide profiling of congenital insulin-like growth factor-1 deficient patients: translational implications in cancer preven- tion and metabolism}

Haim Werner, 1,2

Lena Lapkina Gendler, ${ }^{1}$

Karthik Nagaraj, ${ }^{1}$ Rive Sarfstein, ${ }^{1}$

Zvi Laron ${ }^{3}$

${ }^{1}$ Department of Human Molecular Genetics and Biochemistry, Sackler School of Medicine, Tel Aviv University, Tel Aviv; ${ }^{2}$ Yoran Institute for Human Genome Research, Tel Aviv University, Tel Aviv; ${ }^{3}$ Endocrinology and Diabetes Research Unit, Schneider Children's Medical Center, Petah Tikva, Israel

\begin{abstract}
Laron syndrome (LS) is a rare genetic disorder identified in the $1950 \mathrm{~s}$ by Professor Zvi Laron. LS results from mutation of the growth hormone receptor $(\mathrm{GH}-$ $R$ ) gene, leading to congenital insulin-like growth factor-1 (IGF1) deficiency and dwarfism. Recent epidemiological studies have shown that LS patients do not develop cancer, emphasizing the crucial role of the IGF1 axis in cancer biology. Genome-wide profiling of LS patients conducted in our laboratory led to the identification of genes and signaling pathways that are over- or under-represented in LS compared to healthy controls of the same age range and ethnic group. Differentially expressed genes may be responsible for the association between lifetime low IGF1 values and protection from cancer. This experiment of nature may provide invaluable information that might translate into novel therapeutic approaches in modern oncology.
\end{abstract}

\section{Introduction}

The processes of growth, differentiation and cell death are tightly regulated by multiple cellular and secreted factors that, in a highly orchestrated manner, regulate the time- and tissue-specific expression of a wide array of genes. Disruption of this genetic program may lead to a pathological phenotype, including tumor formation. The vast amount of information that has been generated in recent years following the elucidation of the human genome, combined with the almost daily integration of new data emanating from state-of-the-art technologies, are changing our notions and dogmas about biological processes. In the area of cancer research, in particular, genomic and proteomic approaches, among other sophisticated platforms, are having a huge impact on our understanding of both basic and clinical questions. Biological processes are now amenable for integrative examination at multiple levels of analysis, ranging from molecular to organismal levels. The present review article focuses on the growth hormone (GH)/insulin-like growth factor-1 (IGF1) axis, an important endocrine network with key roles in physiological and pathological states.

\section{The growth hormone-insulin-like growth factor-1 endocrine axis}

The GH/IGF1 axis has a fundamental role in growth and development throughout life. ${ }^{1}$ As originally postulated by Salmon and Daughaday in the mid-1950s, GH actions are mediated by a liver-produced peptide initially termed somatomedin and, subsequently, IGF1. ${ }^{2}$ IGF1 is also produced by extra-hepatic tissues and circulates as a ternary complex with IGF-binding protein3 (IGFBP3) and an acid-labile subunit (ALS). ${ }^{3,4}$ At the cellular level, IGF1 functions as a progression factor that is required by the cell to traverse the cell cycle.

IGF1 and closely related IGF2 ligands activate a common receptor, the IGF-1 receptor (IGF1R), which signals mitogenic, antiapoptotic and pro-survival activities ${ }^{5-8}$ (Figure 1). The IGF1R is an heterotetrameric cell-surface tyrosine kinase receptor coupled to several intracellular second messenger pathways, including the ras-raf-MAPK and PI3K signaling cascades. ${ }^{9}$ IGF1R is vital for cell survival, as illustrated by the lethal phenotype of mice in which the $I G F 1 R$ gene was disrupted by homologous recombination. ${ }^{10}$ IGF1R is evolutionarily related to the insulin receptor (InsR) and is regarded as a key player in malignancy. Transformed cells display augmented numbers of IGF1R on their cell surface as well as increased levels of IGF1R mRNA, suggesting that up-regulation of the $I G F I R$ gene constitutes a common paradigm in most types of cancer. ${ }^{11,12}$ The role of the InsR in cancer biology is still a controversial topic, albeit a number of studies have established that the InsR-A isoform mediates mitogenic actions in breast and other cancers. ${ }^{13}$
Correspondence: Haim Werner, Department of Human Molecular Genetics and Biochemistry, Sackler School of Medicine, Tel Aviv University, Tel Aviv 69978, Israel. Tel: 972-3-6408542 - Fax: 972-3-6405055.

E-mail: hwerner@post.tau.ac.il

Key words: Insulin-like growth factor-1 (IGF1); Growth hormone; Laron syndrome; Cancer protection; Genomic analysis.

Contributions: LLG, HW, data collecting and analyzing; KN, RS, data analyzing; HW, manuscript writing; LLG, KN, RS, ZL, manuscript reviewing.

Conflict of interest: the authors declare no potential conflict of interest.

Funding: this work was supported by a gran from the Israel Science Foundation.

Acknowledgements: the authors wish to thank the Israel Science Foundation for their generous support.

Received for publication: 19 February 2017.

Revision received: 17 March 2017.

Accepted for publication: 17 March 2017

This work is licensed under a Creative Commons Attribution 4.0 License (by-nc 4.0).

(C) Copyright H. Werner et al., 2017

Licensee PAGEPress, Italy

Translational Medicine Reports 2017; 1:6657 doi:10.4081/tmr.6657

\section{Congenital insulin-like growth factor-1 deficiencies: the Laron syndrome case}

Growth retardation in infants is multifactorial, although a large portion of the cases remains idiopathic because no genetic (or other) defect could be identified..$^{14,15}$ Prenatal IGF1 expression is GH-independent, though it becomes reliant on $\mathrm{GH}$ secretion shortly before birth and remains GH-dependent during postnatal life. Congenital IGF1 deficiency is characterized by low serum IGF1 but normal to elevated $\mathrm{GH}$ production. These conditions may result from: i) $\mathrm{GH}$ releasing hormonereceptor $(G H R H-R)$ defect; ii) $G H$ gene deletion (isolated GH deficiency, IGHD); iii) $\mathrm{GH}$ receptor $(G H-R)$ gene deficiency (Laron syndrome, LS); and iv) IGFI gene deletion. Additional conditions leading to congenital IGF1 deficiency are defects of post-GH-R signaling (e.g., STAT5b defects) and $A L S$ mutations. ${ }^{16-19}$

Laron syndrome is a type of dwarfism caused by molecular defects (usually dele- 
tions or mutations) of the $G H-R$ gene, or post-receptor pathways, leading to congenital IGF1 deficiency. ${ }^{20}$ This genetic (autosomal recessive with full penetrance) entity was identified by Prof. Zvi Laron in the late 1950s in three siblings of Yemenite origin and reported in $1966 .{ }^{21}$ The typical features of classical LS are short stature (-4 to -10 SDS below the median normal height), typical face, obesity, high basal serum GH and low IGF1, unresponsive to the administration of exogenous GH. ${ }^{22}$ The recognition that an inherited mutant $G H-R$ gene is the etiological factor behind LS was reported in 1984. ${ }^{23,24}$ Several $G H-R$ defects were identified, including exon deletions and nonsense, frame shift, and missense mutations. ${ }^{25}$ The majority of the mutations are in the extracellular domain of the receptor while a number of mutations were mapped to the transmembrane and cytoplasmic domains $^{26-28}$ (Figure 2).

\section{Laron syndrome patients are protected from cancer develop- ment}

Epidemiological studies have indicated that individuals with increased circulating IGF1 levels, as well as those with insulin resistance and obesity, are at an increased risk for multiple types of cancer. ${ }^{29-33}$ Nevertheless, it is not clear whether IGF1 plays, by endocrine, paracrine or autocrine mechanisms, a role in the etiology or only in the progression of neoplasms. Of basic and translational importance, it is relevant to explore whether individuals with reduced serum IGF1 values have a low cancer incidence.

In recent epidemiological studies including 538 congenital IGF1 deficient patients [230 LS patients, 116 IGHD patients, 79 patients with GHRH-R defects, and 113 patients with congenital multiple pituitary hormone deficiency (cMPHD)] and 752 of their first-degree family members, prevalence of malignancy was assessed by responding to a questionnaire. ${ }^{34,35}$ None of the 230 LS patients (up to the age of 85) included in this cohort developed cancer and only one out of the 116 IGHD patients had a tumor (Table 1). Among 218 first-degree family members (mostly heterozygotes) 18 cases of cancer were reported $(8.3 \%)$. In addition, five malignancies were reported among 86 siblings $(5.8 \%)$. It is important to emphasize that despite the fact that the total number of patients in these studies was small, these differences were highly significant in statistical terms. Furthermore, the observations regarding cancer protection are supported by animal experiments using the $\mathrm{GH}$ $\mathrm{R} / \mathrm{GH}-$ binding protein (BP) KO (Laron) mouse model. ${ }^{36}$

The finding that congenital IGF1 deficient patients do not develop cancer is of major clinical and scientific value. The interpretation of epidemiological data is consistent with the notion that the GH/IGF1 axis has a fundamental role in predisposing progenitor and somatic cells to malignant transformation. Conversely, congenital IGF1 deficiency might confer protection against future development of cancer. We envision a scenario in which life-long lack of exposure to IGF1 in LS patients activates apoptotic, autophagic and cancer-protecting pathways at the organism level. Studies aimed at identifying some of these protective mechanisms will be described in the next section. Of notice, similar results concerning cancer protection were reported by Guevara Aguirre and collaborators in an Ecuadorian cohort of LS patients. ${ }^{37}$

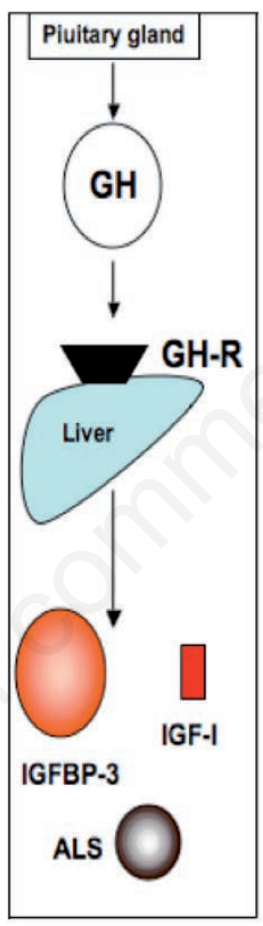

Figure 1. The growth hormone (GH)/insulin-like growth factor-1 (IGF1) signaling pathway. IGF1 and IGF2 bind to the extracellular domain of the IGF1 receptor (IGF1R) and induce autophosphorylation of its tyrosine kinase (TK) domain. The bioavailability of the ligands is controlled by a family of IGF-binding proteins (IGFBP). IGFBP3, the most abundant BP in serum, circulates as a ternary complex with the ligand and an acid-labile subunit (ALS). Inset: activation of liver GH-receptor (GH-R) by GH leads to biosynthesis of IGF1, IGFBP3 and ALS. The GH-R gene is mutated in Laron syndrome.

Table 1. Prevalence of malignancy in Laron syndrome patients.

\begin{tabular}{|c|c|c|c|c|c|c|c|c|}
\hline \multicolumn{3}{|c|}{ Laron syndrome patients } & \multicolumn{3}{|c|}{ First degree family members } & \multicolumn{3}{|c|}{ Siblings only } \\
\hline $\mathrm{N}$ & Malignancies & $\%$ & $\mathrm{~N}$ & Malignancies & $\%$ & $\mathrm{~N}$ & Malignancies & $\%$ \\
\hline 230 & 0 & 0.0 & 218 & 18 & 8.3 & 86 & 5 & 5.8 \\
\hline
\end{tabular}




\section{Genome-wide profiling of Laron syndrome patients}

In order to identify differentially expressed genes that might be linked to cancer protection in LS patients, our laboratory has recently conducted a genome-wide profiling based on our collection at the National Laboratory for the Genetics of Israeli Populations (Tel Aviv University, Israel). ${ }^{38}$ Specifically, RNA was obtained from Epstein-Bar virus-immortalized lymphoblastoids derived from four female LS patients and four controls of the same age range (LS, $44.25 \pm 6.08 \mathrm{yr}$; controls, $51.75 \pm 11.3 \mathrm{yr}$; mean $\pm \mathrm{SD}$; $\mathrm{P}$ value $=0.29$ ) and same ethnic origin (Iraq, Iran, Yemen). One-way ANOVA was performed using Partek Genomics Suite to create a list of differentially expressed genes. A cluster analysis of differentially expressed genes is depicted in Figure 3A. Thirty-nine annotated genes that were differentially expressed in LS compared to healthy controls were identified (with a $\mathrm{P}$ value of $<0.05$ and fold-change difference cutoff $>|2|)$. As shown in Figure 3B, Principal Component Analysis (PCA) revealed a good discrimination between experimental groups. Bioinformatic analyses aimed to identify genes and pathways that are under- or overrepresented in LS are presented in the next section.

\section{Functional analyses}

Functional analyses were conducted to identify co-expressed genes sharing the same pathways. Analyses provide evidence for a number of shared pathways, including cell adhesion, G-protein signaling pathway, cell migration and motility, immune response, Jak-STAT signaling, apoptosis, metabolic pathways, etc. (Figure 3C). This differential expression may, potentially, explain the evasion of LS patients from cancer. Of relevance, bioinformatic analyses detected markedly reduced levels of gene transcripts associated with oncogenic transformation and cell cycle progression. These genes include, among others, cyclin A1, cyclin D1, serpin B2, versican and zinc finger protein $\mathrm{Sp} 1$. Taken together, data are consistent with the concept that life-long lack of exposure to circulating IGF1 in LS patients might lead to downregulation of genes with a positive impact on proliferation and mitogenesis. It is reasonable to assume that IGF1 exposure activates epigenetic and transcription pathways critical for gene expression. Lack of exposure to physiological IGF1 levels in LS patients abro- gates these signaling pathways, with important consequences in terms of cancer avoidance.

\section{Identification of novel metabolic genes in Laron syndrome patients}

Of particular interest, genome-wide profiling revealed enhanced expression of genes associated with protection from toxic xenobiotic substances and metabolites in LS-derived lymphoblastoid cells. These genes include, among others: i) uridine diphosphate (UDP) glycosyl transferase gene family (UGT2B15, UGT2B17; foldchange=12.4); ii) ZYG-11 family member A (ZYG11A; fold change $=4.2)$; iii) ribosomal modification protein RimK family

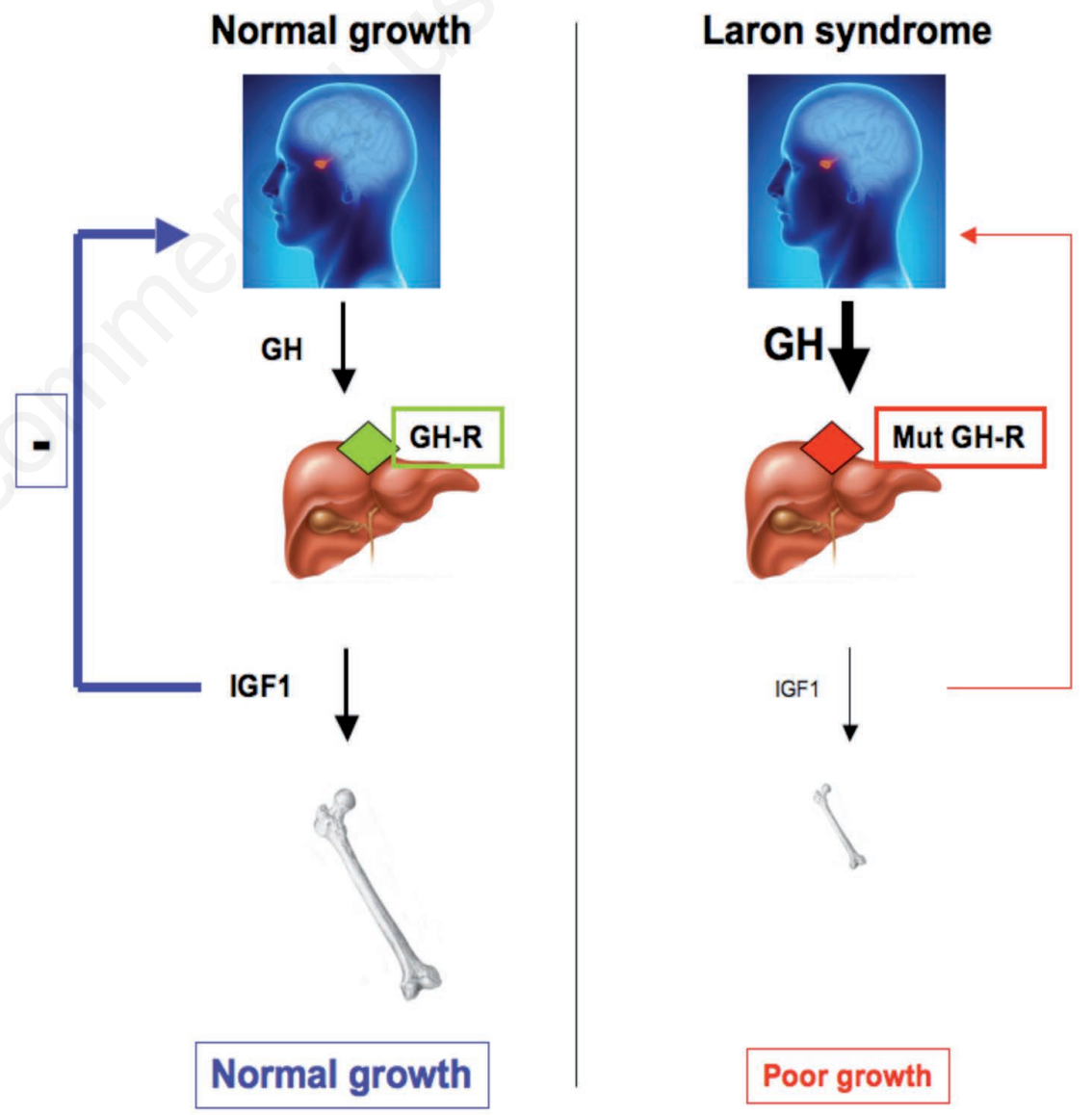

Figure 2. Schematic representation of the growth hormone (GH)/insulin-like growth factor-1 (IGF1) axis in Laron syndrome. Hypophyseal-derived GH stimulates IGF1 production by the liver, with ensuing bone elongation and longitudinal growth (left panel). $G H$ receptor $(G H-R)$ mutations in Laron syndrome lead to congenital IGF1 deficiency, usually linked to poor growth. In addition, abrogation of IGF1 production leads to inadequate negative feedback at the pituitary gland, leading to high circulating GH levels. member B (RIMKLB; fold change=3.7); and iv) thioredoxin-interacting protein (TXNIP; fold-change=2.35). These genes have not been previously linked to the IGF1-insulin signaling pathway.

\section{Uridine diphosphate-glycosyl trans- ferase gene family}

The UDP-glycosyl transferase gene family (UDPGT) plays a major role in the conjugation and subsequent elimination of potentially toxic xenobiotic and endogenous compounds. This protein displays activity towards several classes of xenobiotic substrates, including simple phenolic compounds, flavonoids, antraquinones and certain drugs and their hydroxylated metabolites. Genomic analyses provided UGT2B15/UGT2B17 mRNAs were 12fold higher in LS than in control cells. These results were validated by qPCR. Data

\section{aron syndrome}

Poor growth evidence that the levels of 
is consistent with the finding that survival of LS cells following oxidative damage was several-fold higher than controls. Combined, results imply that increased UGT2B15/UGT2B17 levels in LS might confer upon these cells a protective effect against oxidative and, potentially, genotoxic damage. If substantiated by functional assays, this finding may provide valuable insight into the physiological basis for reduced cancer in LS.

\section{ZYG-11 family member A}

ZYG-11 family member A (ZYG11A) acts as a target recruitment in an E3 ubiquitin ligase complex. The ZYG11A protein is composed of 759 amino acids, includes a leucine-rich repeat and has been postulated to be involved in the ubiquitin-like (Ubl) conjugation pathway. Over-representation of ZYG11A in LS (4.2-fold change) may lead to hyper activation of the Ubl conjugation pathway, with ensuing ubiquitination and degradation of toxic waste compounds.

\section{Ribosomal modification protein \\ RimK family member B}

Ribosomal modification protein RimK family member B (RIMKLB) is involved in cellular protein modification and metabolic processes. It is mainly localized in the cytoplasm, where it displays catalytic and ligase activity. RIMKLB exhibits also metal ion binding activity. Overexpression of this gene in LS (3.7-fold change) may be linked to more efficient catalytic processes, and might be linked to autophagic and apoptotic mechanisms.

\section{Thioredoxin-interacting protein}

Thioredoxin-interacting protein (TXNIP) acts as an oxidative stress mediator by inhibiting thioredoxin activity or by limiting its bioactivity. ${ }^{39}$ TXNIP inhibits the proteosomal degradation of DDIT4 and, thereby, contributes to the inhibition of the mTOR complex. TXNIP belongs to the arrestin family and is downregulated in response to oxidative stress. TXNIP has also been reported to function as a tumor suppressor gene that is commonly silenced by genetic or epigenetic mechanisms in cancer cells. ${ }^{40}$ As mentioned above, genome wide analyses demonstrated that the TXNIP gene was markedly upregulated in LS patients in comparison to healthy control subjects. Considering the fact that IGF1 is upregulated in most types of cancer, we
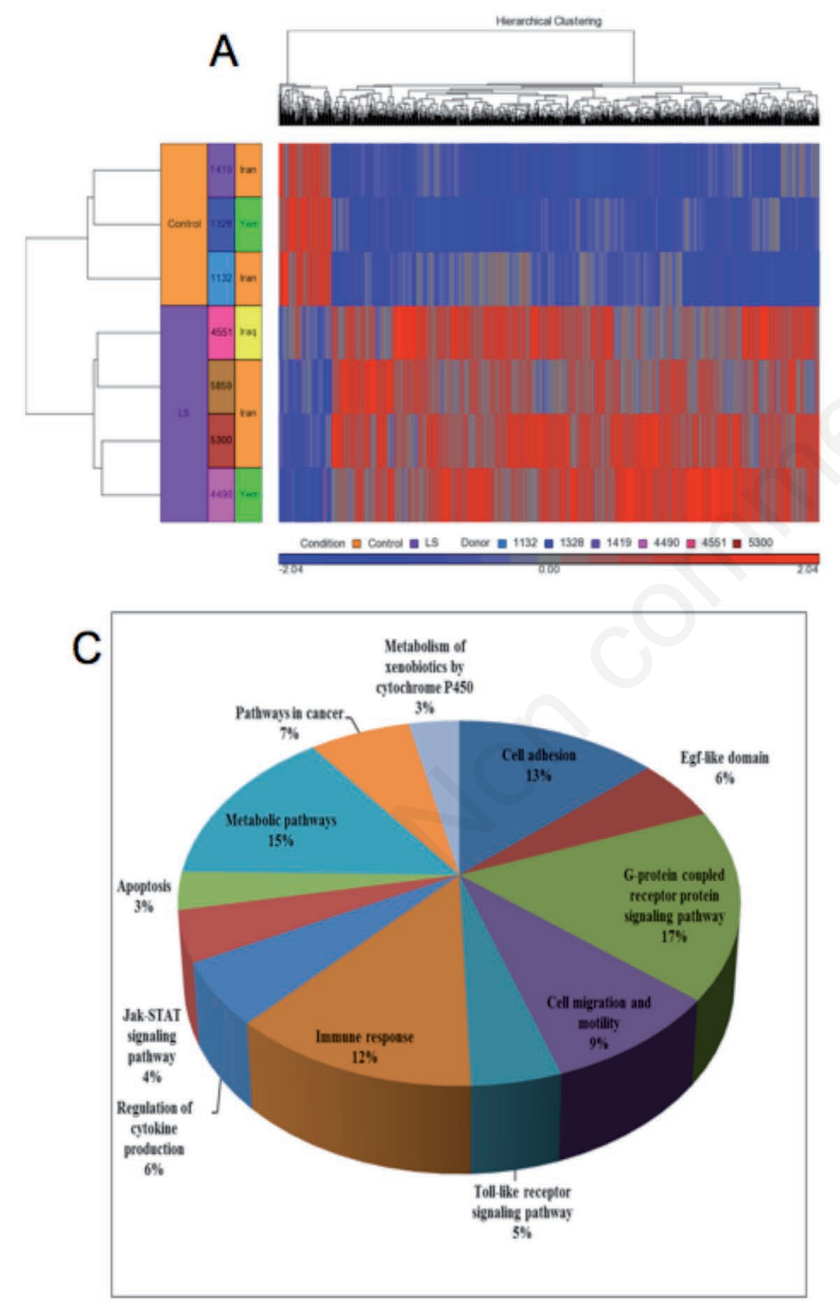

B

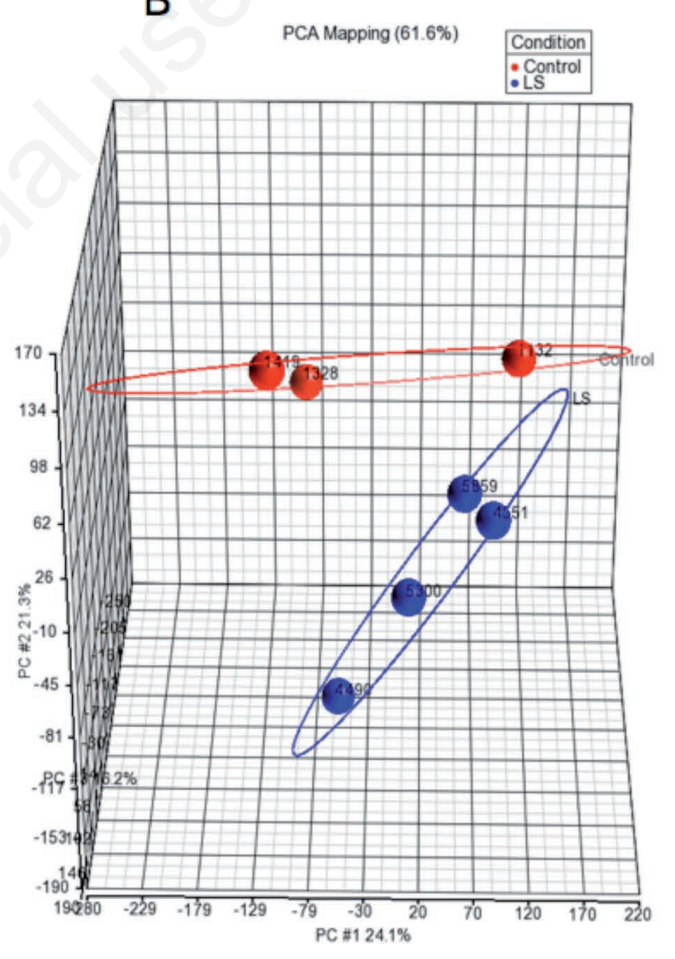

Figure 3. Genome-wide profiling of Laron syndrome (LS) patients. A) Cluster analysis of differentially expressed genes in lymphoblastoids derived from LS patients and healthy controls of the same age range and ethnic origin. The figure depicts a cluster of 39 differentially expressed genes $(\mathrm{FC}>2$ or $<$ than -2 and $\mathrm{P}<0.05)$. Up-regulated genes are shown in red, and down-regulated genes are shown in blue (FC, fold change). B) Principal component analysis (PCA) display of four LS and three control arrays used in the experiment. Blue circles: LS patients; red circles: controls. PCA revealed a good discrimination between both experimental groups. C) Pie chart of gene functions. The sections represent the percentage of genes associated with each function. 
hypothesize that the TXNIP gene may be under inhibitory regulation by IGF1. $T X N I P$, in turn, may act by protecting LS patients from cancer.

\section{Conclusions}

The finding that congenital IGF1 deficient patients do not develop cancer (up to the age of 85) is of an exceptional clinical and scientific value. The interpretation of epidemiological data is consistent with the notion that homozygous congenital IGF1 deficiency, or deficiency in early childhood, may confer protection against future development of cancer. This experiment of nature emphasizes the central role of the IGF1 hormonal axis in cancer and justifies the rational use of available post-genomic technologies in order to elucidate in an unbiased fashion the molecular basis that underlies the evasion of LS patients from cancer. The studies described in this Translational Medicine Reports review identified mechanisms and factors responsible for the association between lifetime low IGF1 levels and protection from cancer.

The results of genome-wide profiling described here shed light on potential genetic changes associated with evasion of congenital IGF1 deficient patients from malignant transformation and may have a major translational impact in oncology. In summary, by mining genomic data from LS patients, a rare condition associated with cancer protection, we might be able to generate clinically relevant novel information and to translate this information into new prophylactic and anticancer tools in oncology. The analyses described here emphasize the power of post-genomic platforms in modern medical research.

\section{References}

1. Rosenfeld RG. The molecular basis of idiophatic short stature. Growth Hormone IGF Res 2005;15(Suppl A):S3-5.

2. Salmon WD, Daughaday WH. A hormonally controlled serum factor which stimulates sulfate incorporation by cartilage in vitro. $\mathrm{J}$ Lab Clin Med 1957;49:825-36.

3. LeRoith D, Bondy C, Yakar S, et al. The somatomedin hypothesis: 2001. Endocrine Rev 2001;22:53-74.

4. LeRoith D, Yakar S. Mechanisms of disease: metabolic effects of growth hormone and insulin-like growth factor1. Nature Clin Pract Endocrinol Metab
2007:3:302-10.

5. Chitnis MM, Yuen JSP, Protheroe AS, et al. The type I insulin-like growth factorI receptor pathway. Clin Cancer Res 2008;14:6364-70.

6. Pollak M. Insulin and insulin-like growth factor signalling in neoplasia. Nature Rev Cancer 2008;8:915-28.

7. Baserga R, Peruzzi F, Reiss K. The IGF1 receptor in cancer biology. Int $\mathrm{J}$ Cancer 2003;107:873-7.

8. King H, Aleksic T, Haluska P, Macaulay VM. Can we unlock the potential of IGF-1R inhibition in cancer therapy? Cancer Treat Rev 2014;40:1096-105.

9. Cox OT, O'Shea S, Tresse E, et al. IGF1 receptor and adhesion signaling: an important axis in determining cancer cell phenotype and therapy resistance. Front Endocrinol 2015;6:106.

10. Baker J, Liu J-P, Robertson EJ, Efstratiadis A. Role of insulin-like growth factors in embryonic and postnatal growth. Cell 1993;75:73-82.

11. Werner H, Bruchim I. IGF-1 and BRCA1 signalling pathways in familial cancer. Lancet Oncol 2012;13:e537-44.

12. Werner H. Tumor suppressors govern insulin-like growth factor signaling pathways: implications in metabolism and cancer. Oncogene 2012;31:270314.

13. Belfiore A, Malaguarnera R. The insulin receptor and cancer. Endocrine Related Cancer 2011;18:R125-47.

14. Rodriguez S, Gaunt TR, Day IN. Molecular genetics of human growth hormone, insulin-like growth factors and their pathways in common disease. Human Genet 2007;122:1-21.

15. Klammt J, Pfaffle R, Werner H, Kiess W. IGF signaling defects as causes of growth failure and IUGR. Trends Endocrinol Metab 2008;19:197-205.

16. Cohen P, Rogol AD, Deal CL, et al. Consensus statement on the diagnosis and treatment of children with idiopathis short stature: a summary of the Growth Hormone Research Society, The Lawson Wilkins Pediatric Endocrine Society and the European Society for Pediatric Endocrinology Workshop. J Clin Endocrinol Metab 2008;93:4210-7.

17. Woods KA, Camacho-Hubner C, Savage MO, Clark AJL. Intrauterine growth retardation and postnatal growth failure associated with deletion of the insulin-like growth factor I gene. New England J Med 1996;335:1363-7.

18. Domene H, Bengolea SV, Martinez AS, et al. Deficiency of the circulating IGF system associated with inactivation of the acid-labile subunit gene. New
England J Med 2004;350:570-7.

19. Kofoed EM, Hwa V, Little B, et al. Growth hormone insensitivity associated with a STAT5b mutation. New England J Med 2003;349:1139-47.

20. Laron Z. Natural history of the classical form of primary growth hormone resistance (Laron syndrome). J Ped Endocrinol Metab 1999;12:231-49.

21. Laron Z, Pertzelan A, Mannheimer S. Genetic pituitary dwarfism with high serum concentration of growth hormone-a new inborn error of metabolism? Isr J Med Sci 1966;2:152-5.

22. Laron Z. Extensive personal experience. Laron syndrome (primary growth hormone resistance or insensitivity): the personal experience 1958-2003. J Clin Endocrinol Metab 2004;89:1031-44.

23. Eshet R, Laron Z, Pertzelan A, et al. Defects of human growth hormone receptors in the liver of two patients with Laron-type dwarfism. Isr J Med Sci 1984;20:8-11.

24. Godowski PJ, Leung DW, Meacham LR, et al. Characterization of the human growth hormone receptor gene and demonstration of a partial gene deletion in two patients with Laron-type dwarfism. P Natl Acad Sci USA 1989;86:8083-7.

25. Ayling RM, Ross R, Towner P, et al. A dominant-negative mutation of the growth hormone receptor causes familial short stature. Nature Gen 1997;16:13-4.

26. Iida K, Takahashi Y, Kaji H, et al. Growth hormone insensitivity syndrome with high serum GH-binding protein levels caused by a heterozygous splice site mutation of the GH receptor gene producing a lack of intracellular domain. J Clin Endocrinol Metab 1998;83:531-7.

27. Duquesnoy P, Sobrier ML, Duriez B, et al. A single amino acid substitution in the exoplasmic domain of the human growth hormone receptor confers familial GH resistance (Laron syndrome) with positive GH-binding activity by abolishing receptor homodimerization. EMBO J 1994;13:1386-95.

28. Shevah O, Nunez O, Rubinstein M, Laron Z. Intronic mutation in the growth hormone receptor gene in a Peruvian girl with Laron syndrome. J Ped Endocrinol Metab 2002;15:103940.

29. Chan JM, Stampfer MJ, Giovannucci E, et al. Plasma insulin-like growth factorI and prostate cancer risk: a prospective study. Science 1998;279:563-6.

30. Hankinson SE, Willett WC, Colditz GA, et al. Circulating concentrations of 
insulin-like growth factor-I and risk of breast cancer. The Lancet 1998;351:1393-6.

31. Renehan AG, Zwahlen M, Minder C, et al. Insulin-like growth factor-I, IGF binding protein-3, and cancer risk: systematic review and meta-regression analysis. Lancet 2004;363:1346-53.

32. Renehan AG, Frystyk J, Flyvbjerg A. Obesity and cancer risk: the role of the insulin-IGF axis. Trends Endocrinol Metab 2006;17:328-36.

33. Calle EE, Kaaks R. Overweight, obesity and cancer: epidemiological evidence and proposed mechanisms. Nat Rev Cancer 2004;4:579-91.

34. Shevah O, Laron Z. Patients with con- genital deficiency of IGF-I seem protected from the development of malignancies: a preliminary report. Growth Hormone IGF Res 2007;17:54-7.

35. Steuerman R, Shevah O, Laron Z. Congenital IGF1 deficiency tends to confer protection against post-natal development of malignancies. Eur $\mathrm{J}$ Endocrinol 2011;164:485-9.

36. Laron Z, Kopchik JJ. Laron syndrome: from man to mouse: Springer-Verlag; 2011.

37. Guevara-Aguirre J, Balasubramanian $P$, Guevara-Aguirre M, et al. Growth hormone receptor deficiency is associated with a major reduction in pro-aging signaling, cancer, and diabetes in humans.
Sci Transl Med 2011;3:70ra13.

38. Lapkina-Gendler L, Rotem I, Pasmanik-Chor M, et al. Identification of signaling pathways associated with cancer protection in Laron syndrome. Endocrine Related Cancer 2016;23: 399-410.

39. Patwari P, Higgins LJ, Chutkow WA, et al. The interaction of thioredoxin with Txnip: Evidence for formation of a mixed disulfide by disulfide exchange. J Biol Chem 2006;281:21884-91.

40. Chen J, Saxena G, Mungrue IN, et al. Thioredoxin-interacting protein: a critical link between glucose toxicity and beta-cell apoptosis. Diabetes 2008;57: 938-44. 